\title{
Backside-surface imprinting as a new strategy to generate specific plastic antibody materials
}

\author{
Gustavo Cabral-Miranda, Magnus Gidlund and M. Goreti F. Sales
}

A backside protein-surface imprinting process is presented herein as a novel way to generate specific synthetic antibody materials. The template is covalently bonded to a carboxylated-PVC supporting film previously cast on gold, let to interact with charged monomers and surrounded next by another thick polymer. This polymer is then covalently attached to a transducing element and the backside of this structure (supporting film plus template) is removed as a regular "tape". The new sensing layer is exposed after the full template removal, showing a high density of re-binding positions, as evidenced by SEM. To ensure that the templates have been efficiently removed, this re-binding layer was cleaned further with a proteolytic enzyme and solution washout. The final material was named MAPS, as in the back-side reading of SPAM, because it acts as a back-side imprinting of this recent approach. It was able to generate, for the first time, a specific response to a complex biomolecule from a synthetic material. Non-imprinted materials (NIMs) were also produced as blank and were used as a control of the imprinting process. All chemical modifications were followed by electrochemical techniques. This was done on a supporting film and transducing element of both MAPS and NIM. Only the MAPS-based device responded to oxLDL and the sensing layer was insensitive to other serum proteins, such as myoglobin and haemoglobin. Linear behaviour between $\log \left(C, \mathrm{mg} \mathrm{mL}^{-1}\right)$ versus charged tranfer resistance $\left(R_{\mathrm{CT}}, \mathrm{U}\right)$ was observed by electrochemical impedance spectroscopy (EIS). Calibrations made in

Fetal Calf Serum (FCS) were linear from 2.5 to $12.5 \mathrm{mg} \mathrm{mL}^{-1}\left(R_{\mathrm{CT}} 1 / 4946.12 \times \log C+1590.7\right)$ with an

$R$-squared of 0.9966 . Overall, these were promising results towards the design of materials acting close to the natural antibodies and applied to practical use of clinical interest.

\section{Introduction}

An antibody $(\mathrm{Ab})$ or immunoglobulin $(\mathrm{Ig})$ is a protein molecule of the immune system that binds with a high degree of affinity and speci? speci?c target species called antigen. ${ }^{1}$ This feature justi?es its intensive use as a reagent in diagnostic assays and therapeutics, with a great impact on the improve- ment of health and welfare in both humans and animals. ${ }^{2,3}$ This is possible due to well-established experimental protocols leading to their production on a high scale, but their inherent complexity and cost $^{4}$ has guided researchers towards ?nding new syntheticpathwaysforAbdesigning.

The most successful synthesis route for obtaining antibodies makes use of molecular imprinting technology, ${ }^{5}$ ?rst discovered in 1931 by Polyakov, ${ }^{6}$ and demonstrated later in 1972 by Wulff and Sarhan. ${ }^{7,8}$ The enthusiasm of researchers within this ?eld of biomimetic materials started then and lasts until today. ${ }^{9}$ In brief, an imprinted polymer is produced by growing a rigid polymeric network around a target molecule. The imprinted sites are generated once the template exits the polymer matrix, theoretically matching the size and shape of the target. Despite many years of progress, targeting big-sized molecules such as proteins by molecular imprinting technology is still a chal- lenge. ${ }^{10-12}$ Size, complexity, conformational ?exibility and solu- bility of proteins are main obstacles, which have been surpassed byusingbulk,epitopesorsurfaceimprintingapproaches. ${ }^{10-23}$

Surface imprinting seems by far the most successful approach in plastic Ab design. It consists mostly in attaching the template to the surface, enabling a pre-polymerization stage where functional monomers are allowed to bind to the template molecule, and polymerize the monomeric material by controlled/living free radical polymerization. ${ }^{17,22,23}$ In general, the covalent immobilization of the template molecules at the surface of solid substrates offers some advantages: it enables imprinting templates that are insoluble in the prepolymeriza- tionmixtureandminimizestemplateaggregation,thusleading

to more homogeneous binding sites. ${ }^{17}$

But the materials produced by surface imprinting contain areas that are able to interact with foreign-proteins by means of non-speci?c interactions, which in turn reduce the selectivity of the material for its targetcompound.Promoting achemical 
differentiation between the binding site and the surrounding surface was recently proposed to reduce this effect. This was made by adding charged positions to the binding sites and polymerizing the surrounding area with neutral monomers. ${ }^{24}$ The obtained biomimetic materials were named Smart Plastic Antibody Materials (SPAMs), showing enhanced affinity and selectivityforthetargeted protein.

However, SPAMs shared a common difficulty in surface imprinting which is the control of the thickness of the polymer layer. When the polymer thickness is higher than the template size, measured when attached to the receptor surface, the template to beimprinted is unable to come out of the polymeric network. Controlling the polymer thickness in surface imprinting is therefore fundamental because the number of binding sites and the dimensions of these sites are directly correlated with thissimplefeature. It is however a difficult task because the protein templates are usually smaller than $10 \mathrm{~nm}$ and the template may be attached to the receptor surface in different positions, which according to the protein conforma- tion above the surface may lead to different polymer thickness requirementswithinthesamematerial.

To overcome the dependency of the polymer thickness, this work reports a backside imprinted layer of the SPAM plastic antibody, this time named MAPS (Materials Antibody Plastic Smart), the backside reading of SPAM. The cross-section of the imprinted material where the protein displays a higher density is the $z$-axis plane corresponding to the proteincovalentbond. If this layer is able to come out, all proteins would be at the same distance for exiting the material. Overall, this new approach is expectedtoensurethatallproteinsareincontactwiththeouter surface and able to exit the imprinted matrix, thus generating surfaces with a high density of rebinding sites.

Herein, this new concept is applied to target oxidized Low- Density Lipoprotein (oxLDL) fractions, composed of different particles of oxidized LDL. Increased plasma levels of oxLDL are associated with atherosclerosis which promotes vascular dysfunction by exerting direct cytotoxicity on endothelial cells and increases chemotactic properties for monocytes and macrophages and consequent transformation into foam cells, which contributes to atherogenesis. The oxLDL level in circu- lating plasma is commonly recognized as an important predictive marker of risk to a cardiovascular event, therefore, novel approaches with simple procedures and low cost are highly urgentandwouldbegreatlyappreciated.

In brief, oxLDL was covalently bond to a supporting ? lm of

carboxylated-PVC cast on a gold support of a screen-printed electrode (SPE), no. 1, let to interact with charged monomers and covered by a neutral polymeric network. The outer surface of this polymer was then covalently attached to another trans- ducing element that was another SPE (no. 2), binding the two SPEs together. These twoSPEs were separated a?]erstripping outSPE-1, whichextracted thecarboxylated-PVC? $\mid$ malong with the oxLDL. The backside of the chemical structure set-up in SPE-1 was now exposed as the top-side of SPE-2. Extensive templateextractionwas further ensured by an enzymatic reac- tion. Non-imprinted materials (NIMs) were also produced as blank and were used as the control for the imprinting process.
All chemical modi?cations of the supporting ?lm and the transducing element were followed by electrochemical tech- niques, both in MAPS and NIM materials. The resulting devices were also evaluated by electrochemicaltechniquesandfurther tested in a Fetal-CalfSerum (FCS) background, nearing its applicationtorealbiologicalsamples.

\section{Experimental section}

\subsection{Apparatus}

Electrochemical measurements were conducted in a potentiostat/galvanostat from Metrohm Autolab/PGSTAT302N with an impedimetric module of the same brand. It was controlled by NOVA so? ?ware. SPEs used were purchased from Drop Sense (DRP-C220AT), having working and counter electrodes made of gold and reference electrode and electrical contacts made of silver. The diameter of the working electrode was $4 \mathrm{~mm}$.

Surface analysis was carried out by using a Scanning Electron Microscope (SEM) of JEOL, JSM-6010LA InTouchScope in vacuum mode. Morphological analysis was conducted using a Scanning Electron Microscope (SEM), JSM-6010LA in vacuum mode. An ultracentrifuge from Beckman, 50Ti rotor, L-8, was used inthepreparation of serumsamples.

\subsection{Reagents}

All chemicals were of analytical grade and de-ionized water (conductivity $<0.1 \mathrm{mS} \mathrm{cm}^{-1}$ ) was used. Poly(vinyl chloride) carboxylated (PVC-COOH, Fluka), 2-nitrophenyl octylether (ONPOE, Fluka), tetrahydrofuran (THF, Aldrich), $\quad \mathrm{N}$-hydroxy- succinimide (NHS, Fluka), $\mathrm{N}$-(3dimethylaminopropyl)- $N^{0}$ - ethylcarbodiimide hydrochloride (EDAC, Sigma), ethylenedi- amine ( $E A$, Merck), vinyl benzoate (VB, Aldrich), (vinylbenzyl) trimethylammonium chloride ( $\mathrm{VB}+$, Acros Organics), benzoyl peroxide (BPO, Himedia), methacrylic acid (MAA, Aldrich), ethylene glycol dimethacrylate (EGDMA, TCI), 3-mercaptopro- pionic acid (MPA, Sigma), proteinase $\mathrm{K}$ from tritirachium album (Sigma), ethyelenodiamine tetraacetic acid (EDTA, diso- dium salt), copper sulphate $\left(\mathrm{CuSO}_{4}\right)$, myoglobin equine skeletal muscle (Myo, Sigma-Aldrich) and human haemoglobin ( $\mathrm{Hb}$, Sigma-Aldrich) were used.

Native LDL was obtained from patients as described later and dialysed prior to its oxidation. oxLDL was obtained by copper based oxidation of LDL and was further analyzed with regard to its MDA content, by measuring thiobarbituric acid reactive substances(TBARS).

\subsection{Solutions}

The buffer used throughout this work was PBS, $\mathrm{pH} 7.2$, and was prepared by mixing $0.9 \% \mathrm{NaCl}, 0.3 \% \mathrm{Na}_{2} \mathrm{HPO}_{4}, 0.2 \% \mathrm{H}_{2} \mathrm{PO}_{4}$ and $0.38 \%$ (w/v) $\mathrm{NaOH}$. For the oxidation of LDL, native LDL from 4 to $6 \mathrm{mg} \mathrm{mL}^{-1}$ prepared in buffer, 20 $\mathrm{mmol} \mathrm{L}^{-1} \mathrm{CuSO}_{4}$ solution in water, and $1 \mathrm{mmol} \mathrm{L}^{-1}$ EDTA in buffer were used.

For the synthesis of MAPS $0.1 \mathrm{~g}$ of PVC-COOH and $0.12 \mathrm{~g}$ of ONPOE dissolved in rv1.5 mL THF were used. For the activation of carboxylic functions $25 \mathrm{mmol} \mathrm{L}^{-1} \mathrm{NHS}$ and $50 \mathrm{mmol} \mathrm{L}^{-1} \mathrm{EDAC}$, both prepared in water, were used. The $4 \mathrm{mg} \mathrm{ml}^{-1}$ oxLDL 
solution used to assemble MAPS was prepared in water. EA solutions were prepared in ionized water with a $0.5 \mathrm{~mol} \mathrm{~L}^{-1}$ concentration. The monomer solutions were prepared in water, having $5.0 \times 10^{-3} \mathrm{~mol} \mathrm{~L}^{-1}$ (VB, MAA or EGDMA) or $1.0 \times 10^{-2} \mathrm{~mol} \mathrm{~L}^{-1}(\mathrm{VB}+)$ concentrations. The radical initiator BPO was

$5.0 \times 10^{-4} \mathrm{~mol} \mathrm{~L}^{-1}$, freshly prepared in water. MPA solution was

$1 \times 10^{-3} \mathrm{~mol} \mathrm{~L}^{-1}$ prepared in ionized water. Proteinase $\mathrm{K}$ solutions were $500 \mathrm{mg} \mathrm{mL}^{-1}$ prepared by suitable dilution of the commercial reagent in buffer. Stock solutions of oxLDL had a $4 \mathrm{mg} \mathrm{ml}^{-1}$ concentration and were prepared in buffer. Less concentrated standards were prepared by accurate dilution of the previous solution in the same buffer. The selectivity study required the preparation of oxLDL, Myo, $\mathrm{Hb}$ and native LDL, all with concentrations of $50 \mathrm{mg} \mathrm{mL}^{-1}$ and prepared in buffer. EIS assays used a solution containing $5.0 \mathrm{mmol} \mathrm{L}^{-1}$ of $\left[\mathrm{Fe}(\mathrm{CN})_{6}\right]^{3-}$ and $5.0 \mathrm{mmol} \mathrm{L}^{-1}$ of $\left[\mathrm{Fe}(\mathrm{CN})_{6}\right]^{4-}$, prepared in buffer.

\subsection{Isolation of native LDL}

Blood samples were collected from healthy volunteers, of both sexes, a aer fasting for 12 hours, with cholesterol and triglyc- eride plasma concentrations ranging within the normal levels (cholesterol $<240 \mathrm{mg}$ $\mathrm{dL}^{-1}$ and triglycerides $<160 \mathrm{mg} \mathrm{dL}{ }^{-1}$ ). These procedures were conducted according to the Ethics Committee on research involving humans of the Institute of Biomedical Sciences, University of $S$ ão Paulo, Brazil, and approved in protocol no. 1033/CEP.

Blood was collected in vacuum tubes (BD Vacutainer), con- taining $0.054 \mathrm{~mL}$ of anticoagulant EDTA, $4.5 \mathrm{~mL}$ volume for suction. The blood of each volunteer was centrifuged $\left(1000 \times g, 4{ }^{\circ} \mathrm{C}, 10\right.$ minutes) separately and later used to create a pool of plasmas. This pool was added with preservatives ( $2 \mathrm{mmol} \mathrm{L}^{-1}$ benzamidine, $0.5 \%$ gentamycin, $0.25 \%$ chloramphenicol,

$0.5 \mathrm{mmol} \mathrm{L}^{-1}$ phenylmethylsulfonyl[uoride and 0.1units per $\mathrm{mL}$ aprotinin), prior to freezing.

LDL (density of $1006 \pm 1063 \mathrm{~g} \mathrm{~mL}^{-1}$ ) was isolated by sequential ultracentrifugation at $100000 \times \mathrm{g}$ for $20 \mathrm{~h}$ at $4{ }^{\circ} \mathrm{C},{ }^{27}$ using (VLDL: $12 \mathrm{~h}, 1.006$ $\mathrm{g} \mathrm{mL}^{-1}$; LDL: $20 \mathrm{~h},<1.063 \mathrm{~g} \mathrm{~mL}^{-1}$;

HDL: $40 \mathrm{~h},<1.21 \mathrm{~g} \mathrm{~mL}^{-1}$ ). LDL was dialyzed for $48 \mathrm{~h}$ in PBS buffer with $0.01 \%$ EDTA and altered (0.22 micron in diameter, Millipore). LDL preparations were stored at $4{ }^{\circ} \mathrm{C}$. The protein content was measured by the Lowry's method. ${ }^{28}$

\subsection{Synthesis of oxLDL and its characterization}

The oxidation of LDL was carried out as described earlier, ${ }^{26,29}$ using native LDL from 4 to $6 \mathrm{mg} \mathrm{mL}^{-1}$. The LDL solution was then incubated in a solution of $20 \mathrm{mmol} \mathrm{L}^{-1} \mathrm{CuSO}_{4}$ at $37^{\circ} \mathrm{C}$. The time of incubation was set to $18 \mathrm{~h}$, as the TBA content (an important indicator of lipid peroxidation) increased up to a maximum and reached a steady value a a er this period of time. In addition, native $L D L$ and oxLDL retention on ion exchange columns (Resource $Q$ ) in the fast protein liquid chromatography (FPLC) have shown increased retention of oxLDL relative to native $L D L$ within this time of oxidation, correlated with an increased number of negatively charged particles. The oxidation of LDL was blocked with the addition of 1 mmol L $\mathrm{L}^{-1}$ EDTA.
The oxLDL solutions displayed nanostructures with an average size of $67.7 \mathrm{~nm}$ f with a standard deviation of $65.6 \mathrm{~nm}$, as determined by DLS measurements using Avid Nano DLS equipment. The expected average molecular weight for oxLDL was $85013.13 \mathrm{kDa}$, corresponding to a polydispersion $\%$ of 0.94 .

\subsection{Synthesis of the plastic antibody material}

The synthesis of the MAPS material was divided into two parts, using two Au-SPEs where only the gold working areas of the electrodes were modi?ed. All solutions were added aller washing out the surface with water or buffer from the previous one. All experiments were conducted at room temperature, except the incubation of oxLDL on Au-SPE/1 that wascarried outat $4{ }^{\circ} \mathrm{C}$.

$\mathrm{Au}-\mathrm{SPE} / 1$ was modi?ed by drop-coating a plasticized PVC- $\mathrm{COOH}$ layer on the gold support. This polymer was then le? to dryfor 1 dayandmodi? byincubation inNHSfor 10 minutes and subsequent reaction with EDAC/NHS solutions for 2 hours. oxLDLsolution was added next and le? for 4 hours. The surface was then le? overnight with the charged monomers. Polymerization was conducted for 5 hours by ?rst adding MAA and EGDMA solutions to the modi?ed support, and then BPO. Finally, the surface was again similarly modi?ed by the same EDAC/NHS reaction, and washed. $\mathrm{Au}-\mathrm{SPE} / 2$ was modi?ed by incubating the Au area with MPA for 20 hours. This new layer was subsequently incubated in NHS for 10 minutes and made react with EDAC/NHS solutions for 2 hours. EA solution was then addedandle? for 1 hour.

The surfaces of SPE/ 1 and SPE/ 2 where then aligned and held together under water for $24 \mathrm{~h}$. Au-SPE/ 2 held the MAPS material that was further treated with proteinase $\mathrm{K}$ for $2 \mathrm{~h}$ and thor- oughly washed with water. NIM surfaces were prepared by using a similar protocol, where the addition of oxLDL andcharged monomers was replaced by buffer/water.

\subsection{Electrochemical assays}

The MAPSs and NIMs were tested by incubating MAPS or NIM devices in oxLDL standard solutions of $2.5 ; 5.0 ; 7.5 ; 10.0 ; 12.5 ; 25.0$; and/or $50.0 \mathrm{mg} \mathrm{mL}^{-}$ 1 . This incubation period was set to 15 minutes. The oxLDL solutions were prepared in buffer or in FCS solution, using these solutions as the blank of the corre-sponding standards.

EIS spectra were recorded in $5.0 \mathrm{mmol} \mathrm{L}^{-1}$ iron redox couple solution. A standard potential of $0.12 \mathrm{~V}$ was applied for this purpose, using a sinusoidal potential perturbation of $0.01 \mathrm{~V}$ and

50 frequencies logarithmically distributed over a frequency range of $0.1-100 \mathrm{kHz}$.

The selectivity of the MAPS surface was tested against native LDL, Myo and $\mathrm{Hb}$ solutions, of $50 \mathrm{mg} \mathrm{mL}^{-1}$ prepared in buffer. The surface was le? in contact with these up to a maximum of $1 \mathrm{~h}$.

\section{Results and discussion}

\subsection{Design of the sensory surface}

The synthesis of MAPS required two different Au-SPEs. Au-SPE/ 1 was used to create theSPAM material, but ensuring that the 


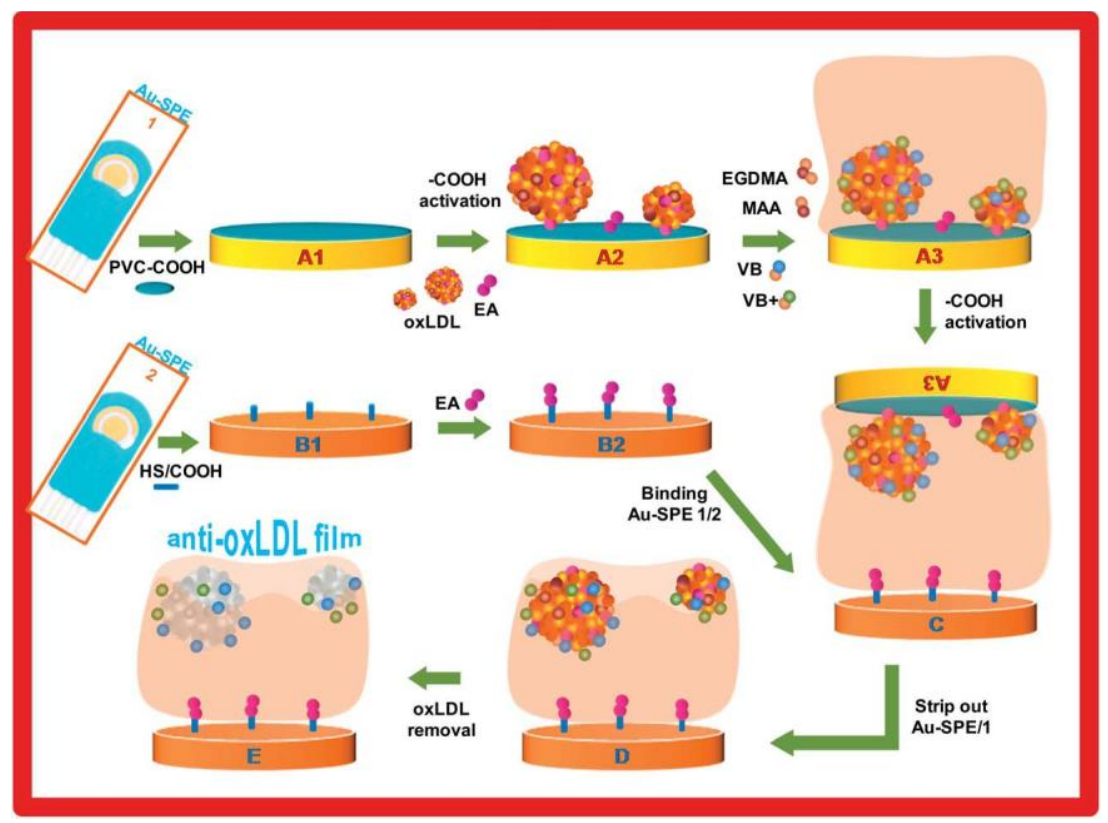

Fig. 1 Assembly of the MAPS material by chemical modification of two separate Au-SPEs.

polymer layer was thick enough to cover the all template compounds present. Au-SPE/2 received this SPAM material in Au-SPE/1, exposing in this way the template layer that was linked to the ?rst SPE, which was indeed the MAPS sensory surface (playing an anti-oxLDL role). All chemical modi?

$\mathrm{Au}-\mathrm{SPE} / 1$ was ?rst coated with a polymeric layer (Fig. 1A1). In order to ensure covalent binding of the oxLDL particles, where many-COOH and$\mathrm{NH}_{2}$ functions are present, this layer could be composed of amine or carboxylic acid functions. But this layer should also ensure a strong bonding to the Au support on one side and to the oxLDL template on the otherside. Athin

? $1 \mathrm{~m}$ of plasticized poly(vinyl chloride) carboxylated (PVC- $\mathrm{COOH}$ ) was found suitable for this purpose. This plasticized polymer showed rubber properties, and remained well attached to the Au working electrode. PVC-COOH solution prepared withouttheplasticizer,o-nitrophenyloctyl ether(ONPOE), was also tested, but the physical bond to the Aulayerwas notas efficient.

The $-\mathrm{COOH}$ groups on top of this layer were subsequently activated for covalent binding of the template. This was done by the wellknown $\quad N$-(3-dimethylaminopropyl)- $N^{0}$-ethylcarbo- diimide hydrochloride (EDAC)/N-hydroxysuccinimide (NHS) chemistry. ${ }^{30}$ This reaction leads to an ester intermediate that reacts promptly with any available amine group in oxLDL. The corresponding reactions may be followedbyanalogyin(ref.31). Thus, once oxLDL was added to the Au-SPE/1 surface, it bound readily to the formerly activated carboxylic groups, thereby forming an amide bond (Fig. 1A2). Ethylenediamine (EA) was added next to deactivate the $-\mathrm{COOH}$ groups that remained activea?er bindingtheoxLDLparticles(Fig.1A2).

The imprinting stage started by introducing charge labels around the oxLDLontheAu-SPE/1 (Fig.1A3). This was achieved by a pre-monomeric arrangement established by means of ionic/electrostatic interactions between the accessible charged sites of the template located on the outer surface and vinyl monomers carrying charged groups. Vinyl benzoate (VB, polar) and VB+ (vinylbenzyl trimethylammonium chloride, positively charged) were used for this purpose. A ?]erthis, thesurfacewas incubated in methacrylic acid (MAA) and ethylene glycol dimethacrylate (EDGMA), to proceed with the polymerization and formation of a rigid and highly reticulated matrix around the template. This polymerization was initiated by benzoyl peroxide (BPO), a radical species, thus avoiding the introduc- tion of unnecessary ionic charged species inside the polymeric matrix. This polymerization reaction was conducted for a long time, ensuring that oxLDL was completely covered by the newly formed polymer. The functional groups at the outer surface of Au-SPE-1 were now -COOR (ester) or $-\mathrm{COOH}$. These groups were activated before binding this layer to the other SPEs, by following the same EDAC/NHS chemistry as previously indicated.

Au-SPE-2 was prepared in parallel, making use of similar chemistry. 3Mercaptopropionic acid (MPA) was placed ?rst on Au. It comprised a three carbon chain with a carboxylic group $(-\mathrm{COOH})$ on one end and a thiol function $(-\mathrm{SH})$ on the other end. The contact between Au and-SH lead to the spontaneous formation of a strong Au-sulphur interaction and the formation of a stable carboxylic layer on the outer surface of the gold support (Fig. 1B1). This surface as further activated by EDAC/ NHS chemistry and added to ethylene diamine, in order to generate an amine layer at the outer surface of the Au-SPE/2 (Fig. 1B2).

$\mathrm{Au}-\mathrm{SPE}-1$ and $\mathrm{Au}-\mathrm{SPE}-2$ were then joined by facing the sensory materials against each other and clipping the SPE supports(Fig.1C). This combination wassubmergedinwater, 
in order to ensure an aqueous environment for the reaction to proceed. At this stage, the activated groups in $\mathrm{Au}-\mathrm{SPE} / 1$ were allowed to react with the amine groups in $\mathrm{Au}-\mathrm{SPE} / 2$, binding the two SPEs together.

Upon separation of the two SPEs, Au-SPE/1 kept the poly- meric layer and most probably part of the oxLDL covalently bound to it, while AuSPE/ 2 exposed a sensing layer where the ox-LDL bound to the polymer ? $1 \mathrm{~m}$ was placed at the same z-level (Fig. 1D). In this separation, it was highly probable that most parts of the template would remain in $\mathrm{Au}-\mathrm{SPE} / 1$, because the polymeric network around it could have prevented the template from coming out connected to the PVC-COOH ? $1 \mathrm{~m}$. Assuming such possibility, complete template removal was granted by incubating the sensing layer of SPE-2 in proteinase $\mathrm{K}$. Proteinase $\mathrm{K}$ cleaved the peptide bonds of the template under mild conditions and subsequently destroyed the polypeptide structure inside the polymer (Fig. 1E). The template was thus removed without disrupting the existing polymeric matrix. The MAPS sensory surface was obtained a? fragments from the imprinted sites by thorough rinsing with buffer and water.

\subsection{Follow-up of the chemical modi?cations on the Au-SPE}

Since the successive chemical modilations of the area of the working electrode changed their electrical features, the assembly of the antioxLDL IIm was followed-up indirectly by monitoring the electron transfer capability of the Au-SPE for the redox pair $\left[\mathrm{Fe}(\mathrm{CN})_{6}\right]^{4-} /\left[\mathrm{Fe}(\mathrm{CN})_{6}\right]^{3-}$. This was done by elec- trochemical impedance spectroscopy EIS, using the Randle's circuit for modelling the experimental data. This circuit was selected for being the simplest and the most frequently employed in gold-based immunosensing. ${ }^{32}$ It comprises the uncompensated resistance of the electrolyte $\left(R_{s}\right)$, in series with the capacitance of the dielectric layer $\left(C_{\mathrm{dl}}\right)$ and the charge- transfer resistance $\left(R_{\mathrm{ct}}\right.$, inversely proportional to the rate of electron transfer), if a redox probe is present in the electro- chemical cell. The two latter components are connected in parallel. An additional component, connected in series with $R_{\mathrm{ct}}$, the Warburg impedance $\left(Z_{\mathrm{w}}\right)$, accounts for the diffusion of ions from the bulk electrolyte to the electrode interface.

EIS data were presented in the form of Nyquist plots (Fig. 2). In this, the imaginary impedance component $\left(\mathrm{Z}^{00}\right.$, out-of-phase) was plotted against the real impedance component $\left(Z^{0}\right.$, in- phase) at each frequency. As expected, these plots showed a semicircle region lying on the real axis at a high frequency range, indicating acharge-transfercontrolled process in which its diameter $\left(R_{\mathrm{CT}}\right)$ equalled the electron transfer kinetics of the redox-probeat the electrodeinterface. This region wasfollowed by a linear behaviour in the lower frequency range, implying a mass-transfer limited process. $^{32}$

The bare gold support of Au-SPE/1 and Au-SPE/2 showed a very small semicircle domain, suggesting a very fast electron- transfer process with a diffusional limiting step (Fig. 2B). The consecutive attachment of the PVC$\mathrm{COOH}$, oxLDL and polymer gave rise to consecutive increases in the electron transfer resistance, resulting in increases in the semicircular section of

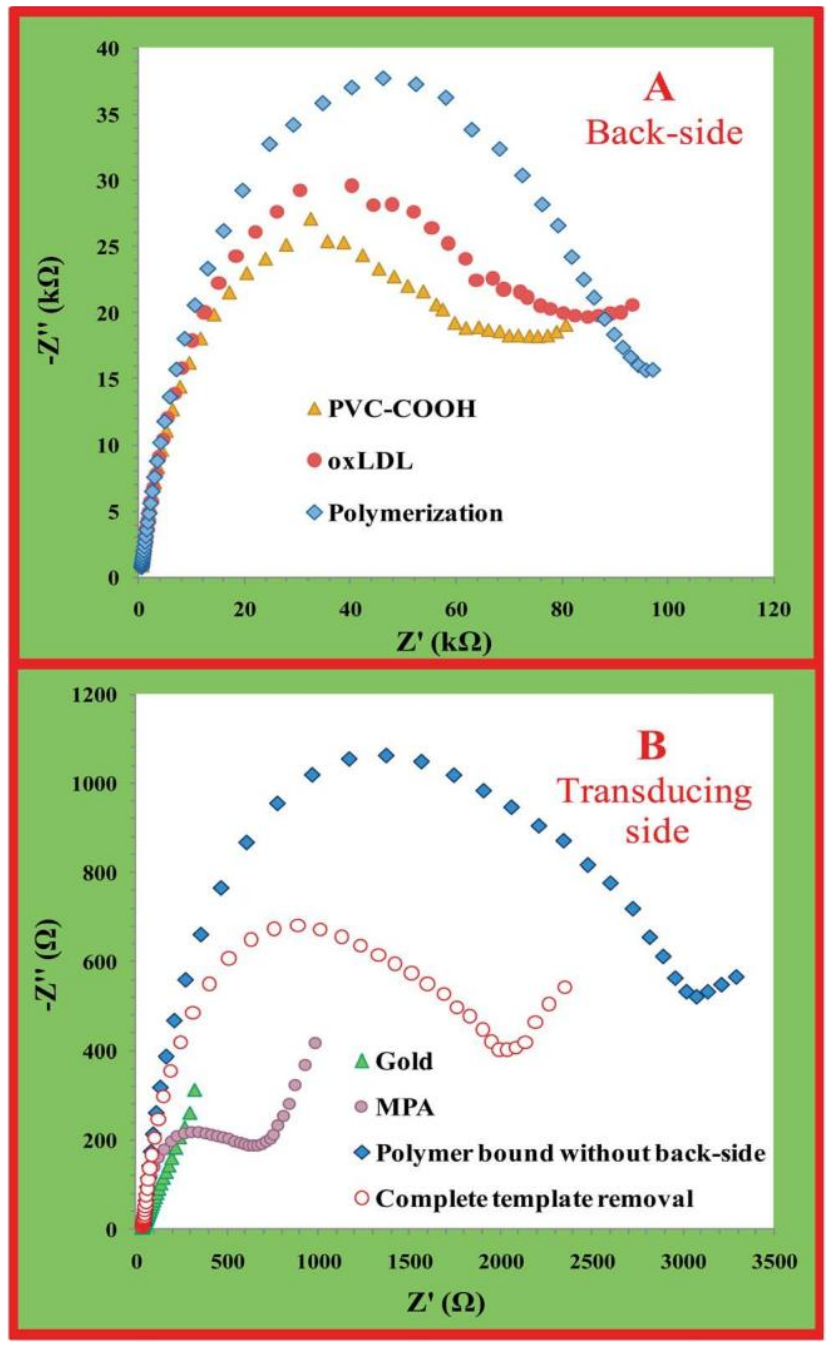

Fig. 2 EIS spectra of the chemical modifications on the supporting film, acting as "backside" of the material (A, Au-SPE/1) and the subsequent assembly of the transducing-side part of the device, including its link to the back-side polymer, back-side removal and complete template exclusion (B, Au-SPE/2).

the Nyquist plot (Fig. 2A). The binding of MPA to the Au in SPE/2 also contributed to a moderate $R_{\mathrm{CT}}$ increase (Fig. $2 \mathrm{~B}$ ), as expectedforthiol compounds of small carbon chains bound to gold. Compared to this, the subsequent binding of the polymer layer formed on SPE/1 promoted a huge increase in $R_{C T}$. This increase was however much smaller than that observed in the original SPE/1, implying that the polymer transfer was not complete. Part of oxLDL was however present within this poly- mer? $1 \mathrm{~m}$ in SPE/2, because the subsequent action of protease decreased the $R_{\mathrm{CT}}$ value. The removal of oxLDLreduced the presence of negatively charged species in the polymeric ? $1 \mathrm{~m}$, which decreased the resistance of a redox probe that was also negatively charged.

The Raman spectra of MAPS and NIM materials were also recorded aiming to ?nd any chemical differences between these. According to the recorded spectra, no differences were observed between the corresponding biosensory surfaces. The obtained spectra are shown in Fig. 3. 


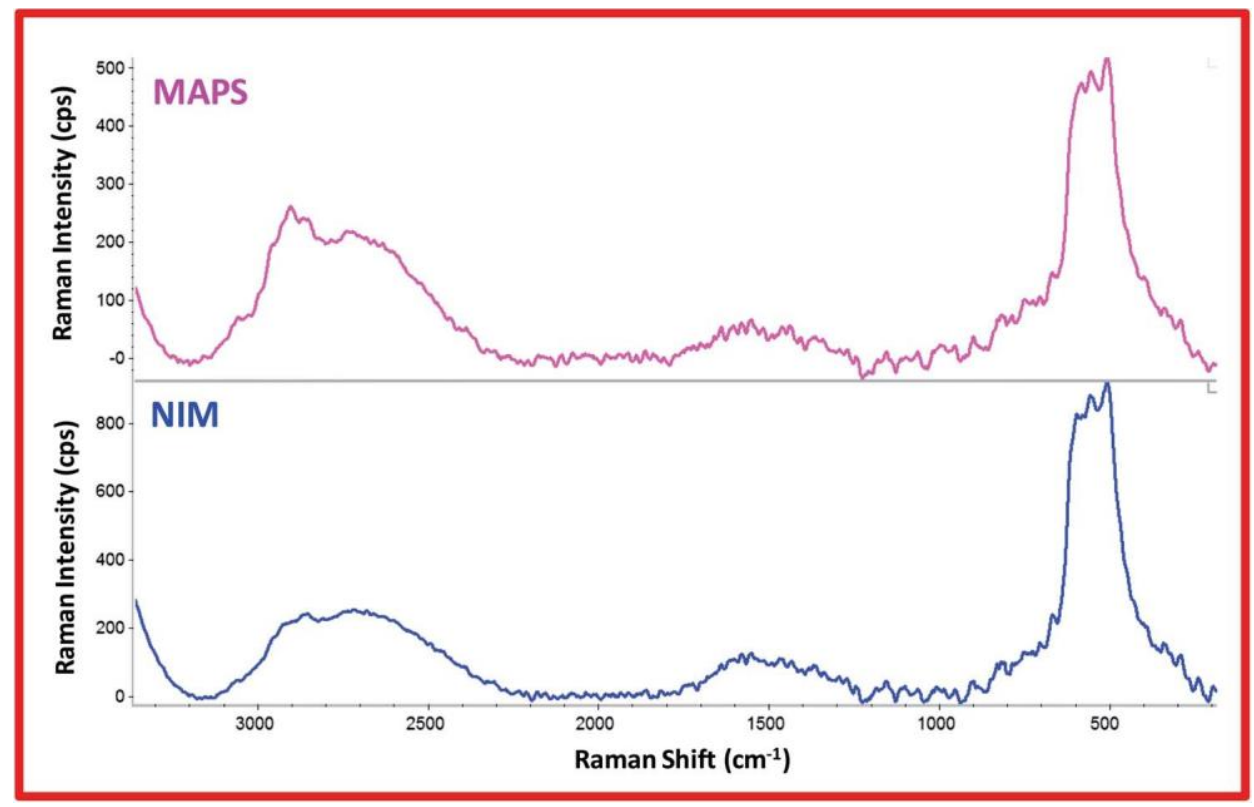

Fig. 3 Raman spectra of the MAPS and NIM material.

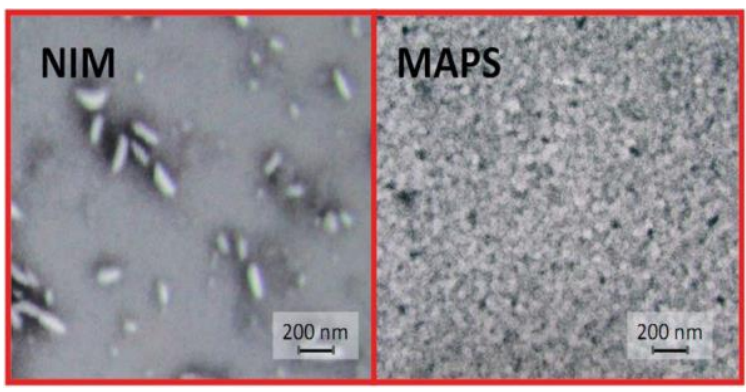

Fig. 4 SEM images of the non-imprinted surface (left, NIM) and imprinted material (right, MAPS).

\subsection{SEM studies}

NIM and MAPS wereobserved byscanning electron microscopy (SEM) and were shown as completely different materials. The obtained images are displayedin Fig. 4.

The MAPS surface showed a high density of "pores" of different sizes (Fig. 4, right). These pores were the oxLDL binding sites, being correlated with the exit of the oxLDL from the polymeric network. This was supported by changes in the poresize, becauseitranged inagreement with the range of sizes of the oxLDL template, as measured by dynamiclight scattering (DLS). The average size was $67.7 \mathrm{~nm}$ fand the standard deviation wasequal to $65.6 \mathrm{~nm}$.

NIM materials showed small agglomerates of the polymer on the surface (Fig. 4, le?). This observation was completely unexpectedand maycorrespond to the presence of $\mathrm{PVC}-\mathrm{COOH}$ residues remaining a? separation of SPE/ 1 andSPE/2.

\subsection{Response of the materials against oxLDL}

The ability of the MPAS surface to interact with the targeted template wasalsofollowedbyEIS. For thispurpose, asolution of $50 \mathrm{mg} \mathrm{mL}^{-1}$ of oxLDL was placed in contact with this type of surface for several periods of time, up to 30 minutes. In general, the $R_{\text {CT }}$ value of the blank surface (incubated in PBS) increased with the presence of 50 $\mathrm{mg} \mathrm{mL}^{-1}$. The maximum

increase in $R_{\text {CT }}$ was observed a? 15 minutes of incubation and the resulting $R_{\text {CT }}$ value remained approximately constant for longer incubation periods. All further studies were con- ducted for a 15 minute incubation period for oxLDL. The typical EIS spectra for PBS and oxLDLsolutionsareseen in Fig. 5A.

The effect of the imprinting process upon the electrical response of the material was further tested by comparing the response of the MAPS surfacetotheNIMone.Unexpectedly, the incubation of the material in PBS or in oxLDL gave rise to the sameelectricalsignal (Fig. 5B). This meant that the NIM surface was unable to establish speci?c/non-speci? interactions with the template. This was mostly surprising because the imprinted template was composed of different species, bearing different sizes. This behaviour indicated the absence of non-speci? binding in NIM surfaces and suggestedaspeci? responsefor the MAPS material.

\subsection{Selectivity study}

The ability of MAPS (plastic anti-oxLDL) to discriminate oxLDL nanostructures from parent or coexisting compounds in serum was checked by incubating the material in native LDL, myoglobin (Myo) and human haemoglobin $(\mathrm{Hb})$. This was done by placing a drop of the corresponding solution on the MAPS surface and incubating it for a suitable period of time. This study was conducted with solutions of $50 \mathrm{mg}$ $\mathrm{mL}^{-1}$ of interfering species, the same concentration as that of the oxLDL solution,

but higher concentrations than those expected in real serum. The time givenforanaffinityreaction withthesensorymaterial 


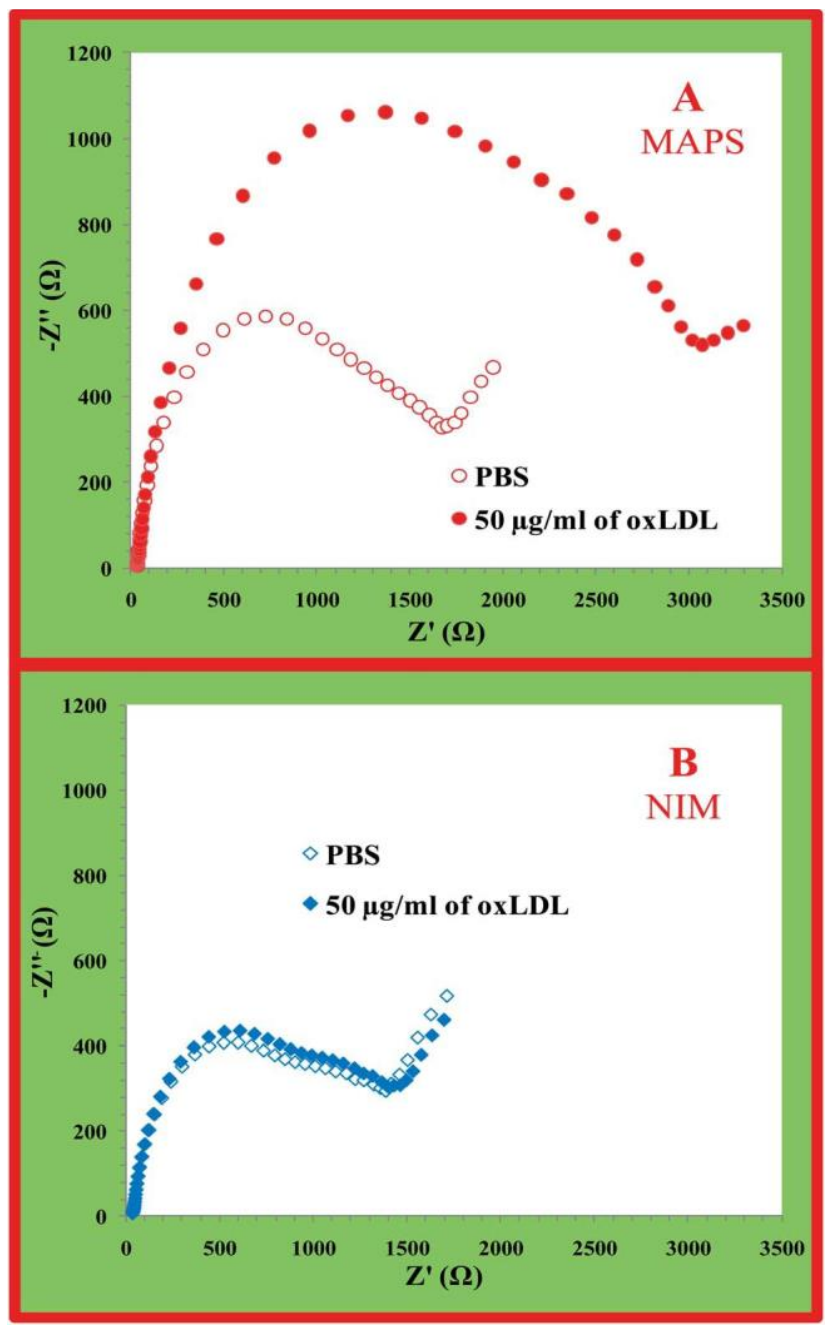

Fig. 5 EIS spectra of the MAPS and NIM with oxLDL solution of high concentration, prepared in buffer.

wassetto1hour, tostrengthenitspossibleinteractionwithany interfering species.

AsmaybeseeninFig. 6,MyoorHbwasunabletochangethe pro?le of the oxLDL EIS spectrum (Fig. 6A and B). This result was extraordinary considering that the imprinted material was highly heterogeneous in size and chemical structure. Further- more, the MAPS surface was unable to reactwithside-proteins, not even by means of non-speci?

The presence of native LDL on MAPS gave rise to a little increase in $R_{\text {CT }}$ (Fig. 6C). Thislittle increasecouldhave been correlated with the high structural similarity between native $\mathrm{LDL}$ and the corresponding imprinted oxidized fragments. However, there is a great chance that this observation re? ?ects the small fraction of oxidized species existing in circulating LDL.

Overall, the obtained selectivity data pointed out the possibility of using the MAPS material as a recognition surface for oxLDL determination in serum, and therefore in patients with normal or overconcentration of the targeted antigens.

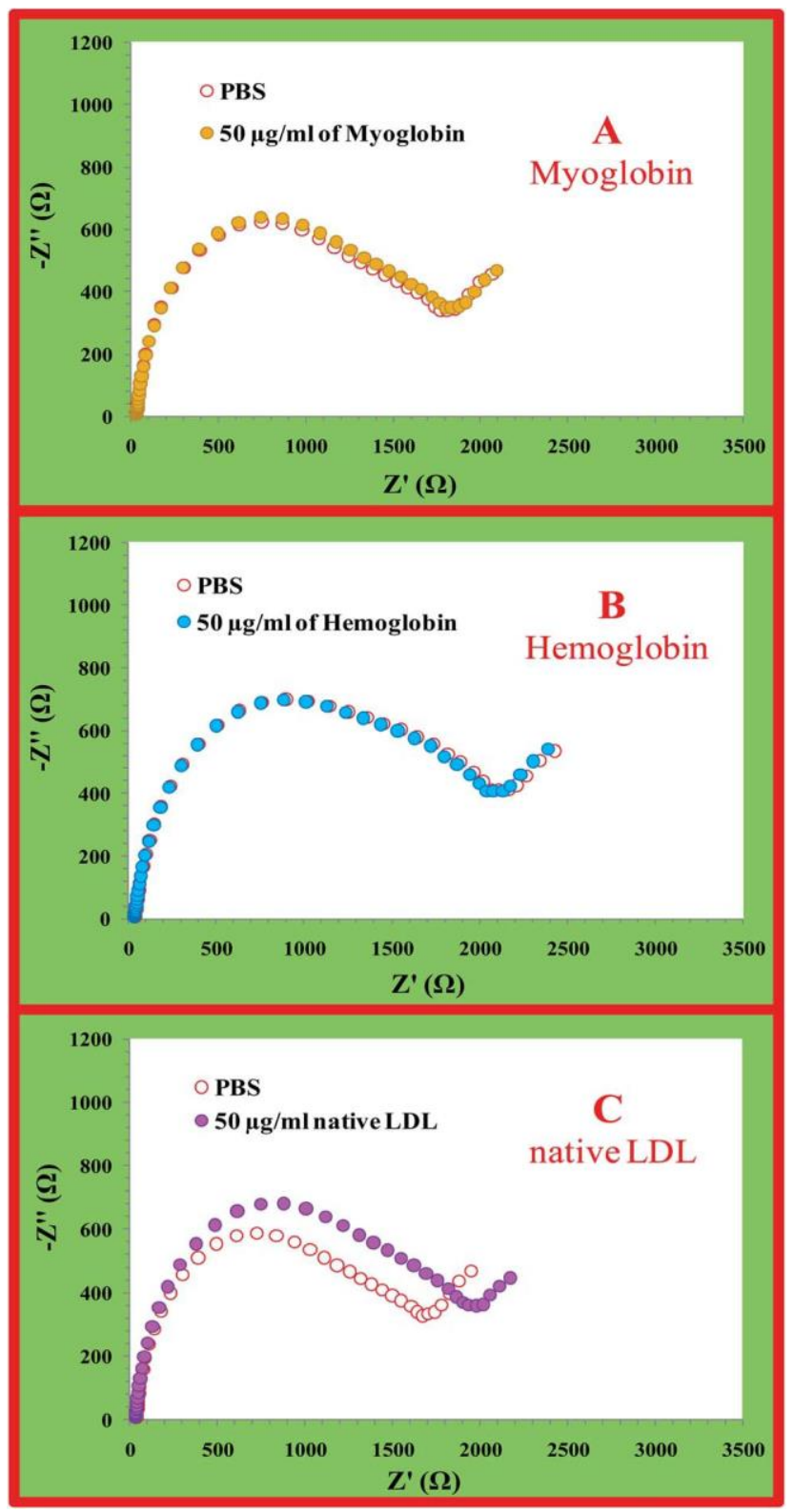

Fig. 6 EIS spectra of the MAPS device in PBS buffer and in different side-biomolecules that mav be present in serum.

\subsection{Sensing oxLDL in serum}

Considering that the anti-oxLDLsurface should operate well in serum to be useful in clinical context, it is important to check the performance of the MAPS under close-to-real conditions. This was done by replacing the buffer in the standard solutions used to calibrate the EIS calibration of the MPAS-device by non- diluted FCS solutions (equal to the biological composition). FCS was used for this purpose because of its great similarity to human serum. ${ }^{33}$

The EIS spectra of the MAPS surface tested with oxLDL in FCS solution showed clear evidence of the presence of oxLDL, leading to a signi?cant increase in $R_{\mathrm{CT}}$. To ensure that this increase in $R_{\mathrm{CT}}$ did not come from FCS, theindividualsignal of 


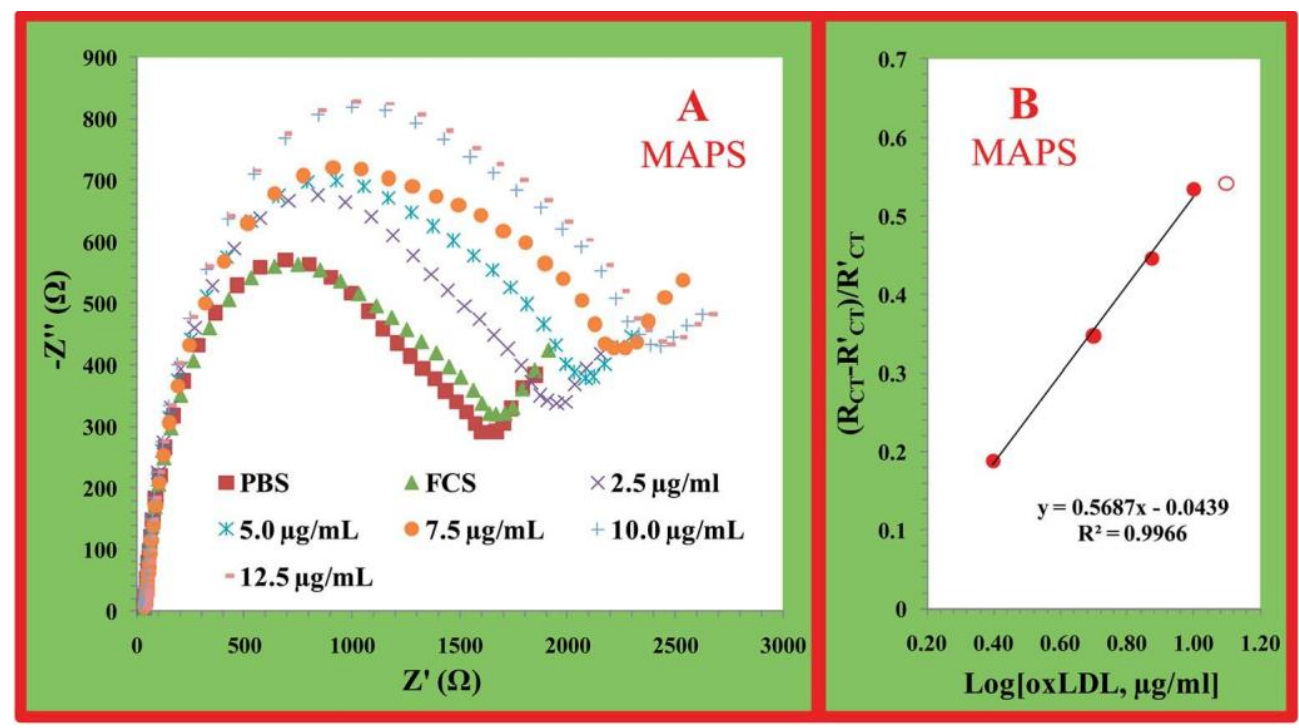

Fig. 7 EIS spectra of the MAPS device tested on an FCS background (A) and the corresponding calibration curve (B), plotting $R_{\mathrm{CT}}$ experimental signals corrected against the signal of FCS in blank solutions.

an FCS solution was measured by incubating the MAPS surface in a solution containing FCS and no human oxLDL $\left(0 \mathrm{mg} \mathrm{ml}^{-1}\right)$. As may be seen in Fig. 7A, only negligible differences were observed between this and PBS. This result is also in agreement with the great selectivity of the material reported in the previous point.

The analytical performance of the immunosensor in FCS solutions was checked by calibrating the device with oxLDL standards prepared in FCS. Since all samples would need at least one dilution step prior to analysis, diluted FCS was used for this purpose. The oxLDL concentration range was changed from $0.0 \mathrm{mg} \mathrm{mL}^{-1}$ (FCS without human oxLDL) up to $50 \mathrm{mg} \mathrm{mL}{ }^{-1}$. The typical EIS spectra are seen in Fig. 7B. The values of $R_{\mathrm{CT}}$ plotted against concentration displayed a curved behaviour, very common to real antigen/antibody interaction and similar to Michaelis-Menten kinetics. For commodity reasons, results were linearized by plotting the logarithm concentration against relative $R_{\mathrm{CT}}$. Relative $R_{\mathrm{CT}}$ was used to correct any differences that may be observed in the active surface of different MAPS- SPEs. The linear portion of the response was observed up to

$10 \mathrm{mg} \mathrm{mL}^{-1}$, and a国er that the material seemed saturated

(Fig. 7B). The linear response in FCS serum, and thus similar to real conditions, was observed from 2.5 to $10 \mathrm{mg} \mathrm{mL}^{-1}$. This region is within the normal range of oxLDL levels, meaning that the presented material offers potential use in human serum having normal and higher levels of oxLDL.

\section{Conclusions}

This novel backside surface imprinting process, where the polymer thickness is no obstacle for the formation of binding sites, was proved successful for targeting a heterogeneous mixture of biomolecules generated bytheoxidation ofLDL.The materialsopreparedseemedtooffer a speci? response for the targeted antigens and a mechanistic response close to biological systems. In general, the chemical functions that were used to assemble the binding sites are different from the ones used to assemble the polymeric network around the template, leading to different electrostatic environments inside and outside the binding position. Inside the binding sites, there are positive charges and polar functions that are expected to be exposed and to interact directly with the template. So, the interaction of oxLDL with the corresponding binding sites is promoted by electrostatic interactions, in a supramolecular arrangement that resembles 'host-guest' electrostatic interactions.

Since the sensory surface is synthetic, it may also be reused to target new concentrations of oxLDL solution, lying in an upper or lower concentration rangethan the onetestedbefore, providedthatthesurfaceis suitablywashed.

Overall, this MAPS concept opens a new path to generate plastic antibodies, creating new expectations in the possibility of generating synthetic materials acting close to monoclonal natural antibodies. The remarkable selectivity observed in MAPS may generate further a new line of devices for diagnosis in point-of-care of different biomolecules of clinical relevance. Ultimately, MAPS sensory materials may replace the use of natural antibodies in conventional diagnosis (mostly ELISA) and contribute to save many animals that are today being used/ sacri?]ced solely for this purpose.

\section{Acknowledgements}

GCM acknowledges CAPES (Coordenação de Aperfeiçoamento de Pessoal de Nível Superior, Brazil) and CNPq (Conselho Nacional de Desenvolvimento Cientíleco e Tecnológico, Brasil, programa Ciência sem Fronteira) for the Enancial support. ScanSci spectroscopic solutions is acknowledged for use of the Avid Nano equipment for Dynamic Light Scattering 
measurements. WITec GmbH is acknowledged for accessing the useofSEM equipmentfromJEOL.

\section{References}

1 M. T. Madigan, J. M. Martinko, D. A. Stahl and D. P. Clark, Immune Mechanisms, in Brock, Biology of Microorganisms, Benjamin Cummings, Pearson Education, 13th Edn, 2012, ch. 30, p. 850.

2 N.S.Lipman,L.R.Jackson,L.J.TrudelandF.Weis-Garcia, ILARJ.,2005, 46, 258.

3 G.K"ohlerandC. Milstein,Nature,1975,256,495-497.

4 A. Bossi, F. Bonini, A. P. F. Turner and S. A. Piletsky, Biosens. Bioelectron., 2007, 22, 1131-1137.

5 N. W. Turner, C. W. Jeans, K. R. Brain, C. J. Allender, V. Hlady and D. W. Britt, Biotechnol. Prog., 2006, 22, 1474.

6 M. V. Polyakov, Zh. Fiz. Khim., 1931, 2, S799-S804.

7 T. Zhu, D. Xu, Y. Wu, J. Li, M. Zhou, T. Tian, Y. Jiang, F. Li and G. Li, J. Mater. Chem. B, 2013, 1, 6449-6458.

8 S.Xu,H.Lu,X.ZhengaandL.Chen,J.Mater.Chem.C,2013, 1, 4406-4422.

9 S. Piletsky and A. Turner, in Molecular Imprinting of Polymers, BiotechnologyIntelligence Unit, LandesBioscience, 2006.

10 N.W.Turner, C. W. Jeans, K. R. Brain, C. J. Allender, V. Hlady andD.W.Britt, Biotechnol. Prog., 2006,22,474-489.

11 T.TakeuchiandT. Hishiya, Org. Biomol. Chem., 2008,6, 2459-2467.

12 M. J. Whitcombe, I. Chianella, L. Larcombe, S. A. Piletsky, J. Noble, R. Porter and A. Horgan, Chem. Soc. Rev., 2011, 40, 1547-1571.

13 S.M. Reddy, G.SetteandQ.Phan, Electrochim. Acta, 2011, 56, 9203-9208.

14 G. Guan, B. Liu, Z. Wang and Z. Zhang, Sensors, 2008, 8, 8291-8320.

15 M. Komiyama, T. Takeuchi, T. Mukawa and H. Asanuma, in Molecular Imprinting, from Fundamentals to Applications, Wiley-VCH Verlag GmbH \& Co. KGaA, 2003.
16 S.Koutsopoulos,Adv.DrugDeliver.Rev.,2012,64,1459-1476. 17 L.Chen,S. XuandJ.Li,Chem.Soc. Rev.,2011,40,2922-2942.

18 N. M. Bergmann and N. A. Peppas, Prog. Polym. Sci., 2008, 33, 271-288.

19 D. S. Janiak and P. Ko] [nas, Anal. Bioanal. Chem., 2007, 389, 399-404.

20 E.Verheyen,J.P.Schillemans, M.vanWijk, M.A.Demeniex, W. E. Henninkand C. F.van Nostrum, Biomaterials, 2011, 32, 3008-3020.

21 A. L. Hillberg and M. Tabrizian, IRBM, 2008, 29, 89-104.

22 A.M.Bossi,P.S.Sharma,L.Montana,G.Zoccatelli,O.Laub and R. Levi, Anal. Chem., 2012, 84, 4036-4041.

23 Z. Lin, Z. Xia, J. Zheng, D. Zheng, L. Zhang, H. Yang and G. Chen, J. Mater. Chem., 2012, 22, 17914-17922.

24 F. T.C. Moreira, S. Sharma, R. A. F. Dutra, J. P. C. Noronha, A. E. G. Cass and M. G. F. Sales, Biosens. Bioelectron., 2013, 15, 237-244.

25 H.Puhl,G.WaegandH.Esterbauer,MethodsEnzymol.,1994, 233,425-441.

26 A. Zaratin, M. Gidlund, P. Boschcov, L. Castilho and E. C. Faria, Am. J. Cardiol., 2002, 90, 651-653.

27 R. J. Havel, H. A. Eeder and J. H. Gragdon, J. Clin. Invest., 1955, 34, 345.

28 O. H. Lowry, N. J. Rosebrough, A. L. Farr and R. J. Randall, J. Biol. Chem., 1951, 193, 265-275.

29 E. C. Fernvik, D. F. Ketelhuth, M. Russo and M. Gidlund, J. Clin. Immunol., 2004, 24, 170-176.

30 K. Y. Jiang, L. S. Schadler, R. W. Siegel, X. J. Zhang, H. F. Zhang and M. Terrones, J. Mater. Chem., 2004, 14, 37-39.

31 F.T.C.Moreira, R.A.F.Dutra,J.P.C.Noronha,A.L.Cunha and M. G. F. Sales, Biosens. Bioelectron., 2011, 28, 243-250.

32 M.I.Prodromidis, Electrochim. Acta,2010,55,4227-4233. 33T. Lindl, Zell-und Gewebekultur, Spektrum akademischer Verlag, Heidelberg, 5th edn, 2002. 